\title{
MANETs as propellant in the growth of the Internet of Things
}

\author{
Aniket Deshpande \\ (Research Scholar, Faculty of Engineering and Technology, Mewar University, India - 312901)
}

\begin{abstract}
As we fast forward towards 2020 world is technologically moving towards the digital life where almost any and every device or object could get some for of IP connectivity and in turn possibly to the Internet. IDC is anticipating more than 28 billion devices on the Internet. With this evolution, Mobile Ad Hoc Networks (MANETS) have now become more critical than ever before - be it the public Wi-Fi hotspots, mobile Internet or simply smart homes. This technology has thus now gathered the attention in this newly emerging digital era to perform varied tasks and to transfer the data without any fixed infrastructure support. Cloud computing is one of its form and this technology moves way farther to benefit the human life. Thus MANET has got renewed focus as being one the key enablers of the Internet of things, a new advancement in order to enhance the overall performance and to provide a more sophisticated solution to the routing networking problem.
\end{abstract}

Keywords: Mobile Ad-Hoc Networks, Internet of Things, TCP, Smart Devices, TCP

\section{Introduction}

The recent advances in the performance of technology, computers and wireless networks are expected to propel the development of new applications for mobile computing, advanced wireless and thus consequently the growth in the use of this Internet of Things. These mobile computing applications have been developed primarily based on TCP/IP and deployed widely on Ad-Hoc Networks. The focus of development made with respect to this type of network has been to support robust and efficient operations, incorporating routing features as well. This also presents greater challenges for wireless communication, in view of the issues related to energy consumption and reliability of links. Inside the Internet community, the technology of "Mobile IP" refers to the routing support for mobile devices. Within this new technological advancement, any and every device, be it Smart Phones, Smart TVs, surveillance devices and possibly any machine or gadgets that human imagination could stretch, is becoming IP enabled and getting hooked onto to an accessible network gateway. This kind of access in today's smart world has potential of being the best possible use-case that an Ad-Hoc Network could ever have. Hence this paper is aimed at determining the necessity of performance and reliability of Mobile Ad-Hoc Networks towards enabling the growth of the Internet of Things. This will be done through a thorough online secondary research in order to complete the essay effectively.

\section{Literature Survey}

A Wireless Network Mobile Ad Hoc system is formed by a set of various nodes through mobile application, able to communicate with their neighbors by diffusion (broadcast) or point-to-point connections, without any fixed infrastructure support. Development of routing protocols for this type of network has several characteristics to be considered: the existence of dynamic topology, the width limited bandwidth of your links and the need for energy conservation. These network systems has a wide usage in industrial applications and trade involving exchange of mobile data cooperatively [1]. When combined properly with the satellite-based information technology, Mobile Ad-Hoc networks can provide convenient flexible ways and efficient methods for the establishment of communicating in fire operations, search and rescue or other scenarios that requires the immediate establishment communication with dynamic survival networks.

Dozens of protocols have been discussed in hundreds of technical articles, and some have been standardized by the MANET working group (Mobile Ad hoc Network), the IETF: AODV, OLSR, TBRPF and DSR (Draft) [2]. The growing interest in this new technology has brought attention of the universities and research centers and the volume of publications available today show the importance of this issue. In accordance with the available routing strategy, these protocols have been classified into proactive, reactive, hierarchical and supported by location (GPS). The Ad hoc Wireless Mobile Network is formed by a combination of nodes through mobile connections, capable of collaborating with their neighbors by broadcast or point-to-point connections, without the need of any fixed supporting infrastructure [3]. 


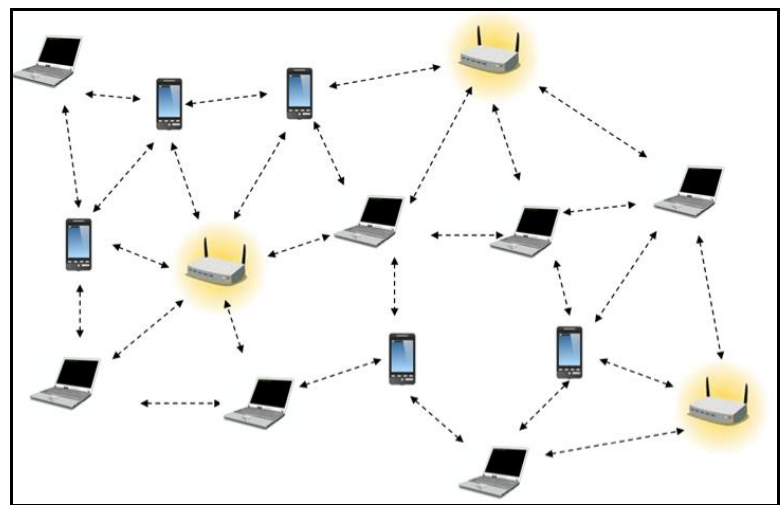

Figure 1. Sample Representation of Mobile Ad-Hoc Networks

The development of routing protocols for this type of network presents several unique characteristics that need to be taken into account: the existence of a dynamic topology, the limited bandwidth of its links and the need for energy conservation [4]. Ad-Hoc mobile networks are widely useful in industrial and business applications involving collaboration via mobile date. When sufficiently pooled with satellite-based information distribution, the technology of mobile ad hoc networks may offer an enormously supple and well-organized technique for the founding of communications in fire control, saving and rescuing operations, or other scenario unique demands the immediate communication with dynamic networks of survival.

Internet of Things (IoT) denotes to the diverse visible embedded connections that aids in computing like the system commonly used as built-in sensor deployed in the vehicle, monitoring heart implantation, biochip transponders on grange faunas. Thousands of campaigns interrelate with other via wireless/wired statement without human involvement. In other exploration IoT definitely serves as new means with the help of which maneuvers can connect with each other using sensors. IoT has been deployed in the numerous use-cases shown in below figure, to regulate many things employed in our day-to-day life such as automobile, construction, application, etc.

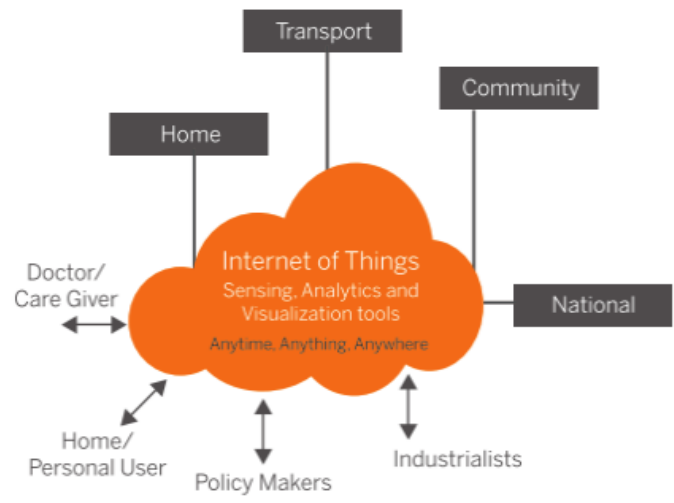

Figure 2. Conceptualization of Internet of Things

\section{Contribution Of TCP And MANET In Spread Of IOT}

TCP has been designed and optimized for over 20 years as a mechanism for wired networks enabling the efficient use of the available bandwidth among various connections contending for the same resources. However, in designing the control mechanisms of flow and recovery of TCP, errors introduced owing to the wireless link characteristics are not accounted for and have disseminated on the Internet over all these years. For example, TCP takes the assumption that all the packet level losses are result of the congestion in the network. If sending TCP experiences the loss of a packet, in any case reduces the pace of transmission halving the value of the transmission window halving the value of the transmission window, thus achieving lower performance.

The use of Wireless Networks on the Internet has undergone considerable transformation in the last 10 years due to ever evolving development and dissemination of wireless technologies [5]. The number of mobile Internet users is exponentially increasing with estimates of over 20 billion users worldwide. It is therefore necessary to study the impact of the difference that these wireless technologies have on various layers of TCP/IP stack and more particularly on the transport layer. This technology was an evolution of Networks Packet Radio via Mobile (Mobile Packet Radio Networking) and Networks Mobile mesh (Mobile Mesh Networking). This type of network has no fixed station, all its nodes can move freely and dynamically connect to an arbitrary way. 
Responsibility for organization and control of the network is distributed among all of its nodes. Mobile Ad-Hoc Network, as per the conventional definition, is "an autonomous system of mobile devices (also serving as routers) connected by wireless links, the union of which form a communication network modeled as an arbitrary graph." These mobile devices can be simply located on aircraft, ships, trucks, cars, or even hand-held by humans [6]. The more applications highlighted in the literature for this type of the network are the military areas wherein at a battlefield, for example, the tanks may want a battalion to establish an immediate communication in a hostile and noisy environment, with no time or resources to create a wired infrastructure. Other possible applications would be in rescue missions arising from natural disasters; commercial and educational applications and sensor networks. According to the definition presented last in a mobile ad hoc network, also known as a peer-to-peer network, there may be one or more pairs of nodes who cannot communicate directly, being an out of range of the other. Still, the communication between these two nodes may occur, but in this case, with help us intermediaries who are willing to make the relay (relaying) of posts. In general, all the nodes of these functioning network as routers that determine and preserve routes to other nodes on the grid. In other words, for two specific hosts on a MANET to interoperate, multiple hops comprising of mobile ad-hoc nodes may be needed, and this interoperability is also dependent on the number of hops and the number of intermediate nodes used to facilitate communication between those two hosts [7].

This type of network requires that packets of information be transmitted via store-and-forward method, from the origin to an arbitrary recipient through the intermediate nodes. When we change, the resulting effect in the network topology must be informed to all the other nodes, such that the topology information may be updated permanently. The networks are tolerant of failures and withdrawal of intermediary stations because the overall organization and control does not depend only on the ones we determined. This is because all of its nodes have the function of making routing and packet retransmission. In the same fashion, new nodes can be added easily to these networks. This concept contrasts with the cell networks using the network model simple jump (single hop), that is, between the source and destination devices, there is only base station that operates as a fixed access point. In these networks, communication between two mobile nodes depends completely on the backbone wired and fixed base stations. In a mobile network, wireless ad hoc, on the other hand, there is no infrastructure and network topology may change dynamically, in a non- predictable way, since the nodes [8].

Basic characteristics of Mobile Ad-Hoc Networks, as seen from the topology may vary depending on the movement of its nodes and adjustments to their parameters of transmission and reception. The areas of scope or coverage can be changed anytime. In addition, networks mesh-based furniture can be used as alternatives or as solutions robust and cheap to use infrastructure cell-based mobile networks. When combined properly with the delivery of satellite-based information technology, mobile Ad-Hoc Networks can deliver a tremendously supple technique for establishing communications for fire operations, search and release or other situations that require a quick and immediate establishment of communication with networks [9].

\section{Criticality Of TCP In Optimal Performance Of Manets}

A mobile node can be physically and directly connected to an external subnet of some fixed network or be connected via a wireless connection, dial-up, etc. Support for this form of mobility requires improvements in management addresses and interoperability protocols, among others. Mobile IP technology implements support for the travelers, as follows: when a node moves out of your home network, making it a traveler node in the external network (roaming), it reports to a node of your home network, called agent relay (home agent), the packets addressed to it must be retransmitted to a given node from the external network, called agent receiver 8 (Foreign agent), that network [10]. Then it registers itself in the receiving agent for all packets addressed to him be redirected through the relay agent to your home network to the agent receiver that delivers the traveler node. When the traveler node returns to its home network, it informs both nodes (agents) involved in the redirection of the packets that it is the receiving node is that has the responsibility to "deliver" the packets to the visitor node.

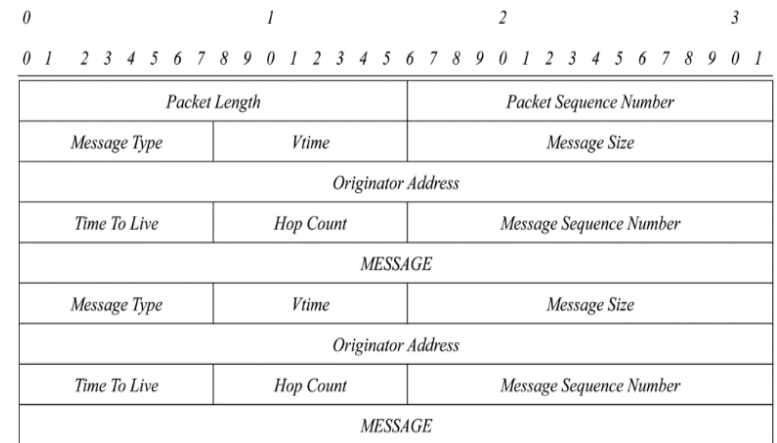

Figure 3. Sample format of Messages transmitted via Mobile IP 
It is worthwhile to note that other nodes in any network external, need not be informed of the movement of the traveler node, since the agents relay and the receiver guarantees the delivery of packets throughout the roaming process. In the structured wireless network, the nodes most suitable for functions of relaying and receiving are not necessarily the access points of the origin cell and the destination node traveler, since no other nodes can take on these tasks. In a mobile Ad-Hoc Network there is no concept of relay agent, since all nodes are mobile and can move freely. The core network functions generally use routing protocols traditional known, which implement routing techniques called vector distances (distance-vector or hop-by-hop) or status of links (linkstate), used in operation of wired networks [11]. Unlike this type of solution, the goal of MANET is to extend the concept of mobility to allow mobile wireless areas, completely autonomous, comprising a set of nodes (which may be combined stations and routers) form an Ad-Hoc network routing infrastructure amongst themselves.

The routing in MANETs is intrinsically different from traditional routing used in existing infrastructure networks mainly due to the limitation of resources, especially with respect to bandwidth and autonomy drums. Still, the number of nodes in MANETs is generally much lower than that of wired networks. Thus, the issue of scalability routing protocols for multi-hop wireless networks can be impacted by excessive overloads routing messages caused by population growth, and the mobility network. In this type of network, routing protocols depend on several specific factors such as topology, the initialization method of the request, the choice of routers to be used (intermediate nodes) as well as specific features of the location of use, that can possibly serve as a heuristic for determining the path faster and more efficient to the target node [8]. As seen, the routing between two specific nodes may depend on the service packet forwarding provided by other intermediate nodes, called nodes' jump (hop nodes), responsible for the routing of packets between source and destination. As the jump we can also move freely, the routing path considered optimal at the time of forwarding a packet may contain jump us that will not be part of the optimal routing path in the next moment.

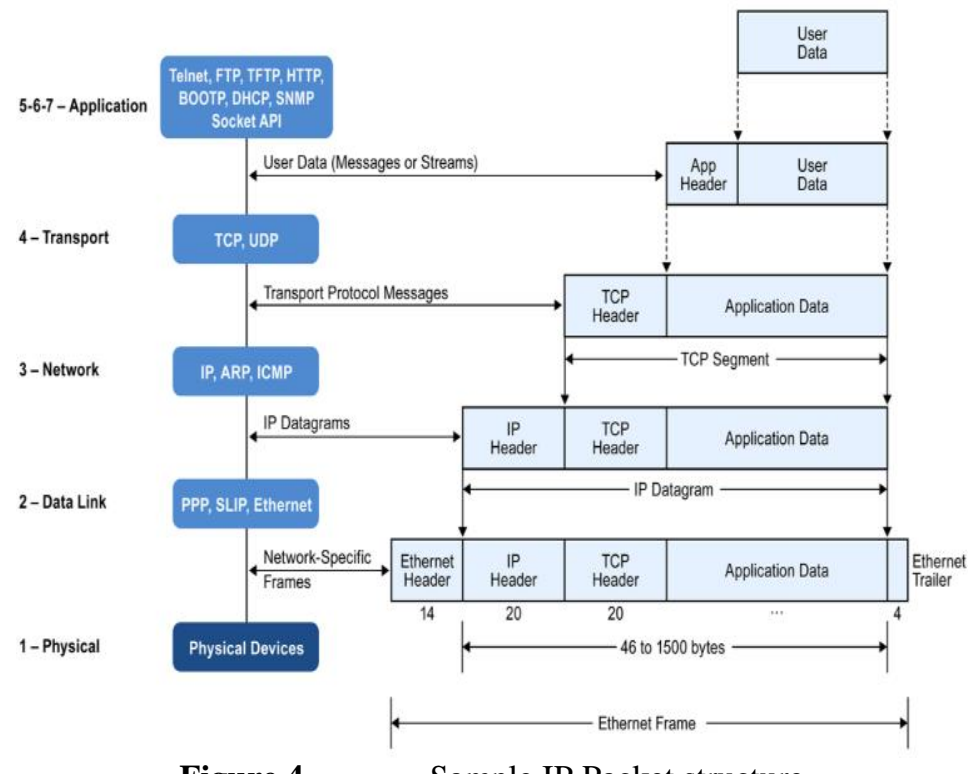

Figure 4. Sample IP Packet structure

Also, a given moment may not even exist paths between those same two nodes of origin and destiny. The main aspects to be considered in the study of renewed focus on performance of TCP in MANETs towards the success of the Internet of Things include reliability, availability and performance of the network. The reliability refers to the study of metrics related to error occurrences, transmission, delivery of packets out of order, delivery of duplicate packets, etc. The determining the availability effective network considers the metric for the study rated capacity of the network to the average time values of error occurrences and Median time to recovery of these errors, etc. [4]. The performance of the network is calculated from metrics related to the number of packets correctly transmitted per unit time, the consumption of network resources in sending the control messages (network management, synchronization and authentication between stations, establishment and maintenance of routes), the response time variability, etc. Knowing the applications used on the network is also crucial to determine the set of metrics to consider in the analysis of the network performance measures. Unlike this type of solution, Mobile Ad-Hoc Networks extend the concept of mobility domains to allow mobile wireless sets consisting of fully autonomous form themselves, an Ad-Hoc type routing infrastructure type.

The development of routing protocols for such type of networks need main focus to enable the support for a robust and efficient operation and, therefore, have to incorporate the routing functionality within the mobile 
nodes themselves. This demand also presents greater challenge for wireless communications because its nodes (to be routers and combined stations) have quite limited resources mainly with respect to bandwidth and battery life which makes the solutions intrinsically different routing used in the existing infrastructure networks [1]. Another aspect to consider is that the number of nodes in a Mobile Ad-Hoc Wireless Network is generally much less when compared to the wired networks and, accordingly, the scalability of routing protocols for multi-hop wireless networks can be impacted by excessive overloading routing control messages caused by this growth in the numbers of nodes and the network mobility. In this type of network, routing protocols depend on several factors specific, such as the topology, boot process, the request, and the choice of the routers (Intermediate nodes) to be used, as well as the location of the nodes. These factors may serve as a heuristic to determine the fastest and the most efficient route to the destination node.

As we have seen earlier, when two specific nodes want to communicate and do not have a direct link to each other, communication between both depends on the service routing provided by intermediate nodes, responsible for the routing of packets between source and destination. As the packets can also move freely between the nodes, the routing path considered optimal in forwarding a packet may contain jump of nodes that will not be part of the routing path. Still, in a given moment, might not even exist paths between those same two nodes of origin and destination [4]. This makes clear the requirement of difference between the routing protocols in this new scenario and the wired networks. Proactive routing protocols have a characteristic in common: Routing information is exchanged permanently between network nodes, regardless of the occurrence of requests. This type of protocol has properties that are very important for real-time applications or requiring guarantee, such as network monitoring, access to low-latency routes and support alternative paths. An example of this type of protocol is the DSDV (Destination-Sequenced Distance Vector - Target Distance Vector in Heels) that implements and improvement in traditional technique distances vector to ensure no loops (loops) [11]. To prevent loops, the destination node provides and marks each route with a number of sequences. To maintain consistency of routes, routing table updates are periodically transmitted (proactive protocol).

\section{Future Trends In IOT}

According to Cisco Systems in 2008, there were more things connected to the Internet on planet earth than humans. By 2020, 50 billion things must be connected by means of sensors and communication networks, most wireless. Among these connections, real- time data flow that can be used for various purposes. The first users of the IT have the focus on inventory control and supply chain management systems to identify, store, and track their products in a unique way and in real time. However, according to The Economist, $75 \%$ of global business leaders are exploiting the economic opportunities of the Internet of Things to create new markets or enhance existing services. GE estimates that it has the potential to add a value of 10 to 15 trillion dollars to the Gross Domestic Product (GDP) over the next 20 years, overtaking the US economy. While the potential of it is difficult to be ignored, it is also important to emphasize that it is not as simple as pasting sensors connected to the Internet at all. We could, for example, put a sensor connected to the internet in a 1999 model car, but he did not provide the driver nothing but the use of GPS on your smartphone to help you find the spot where he parked [9].

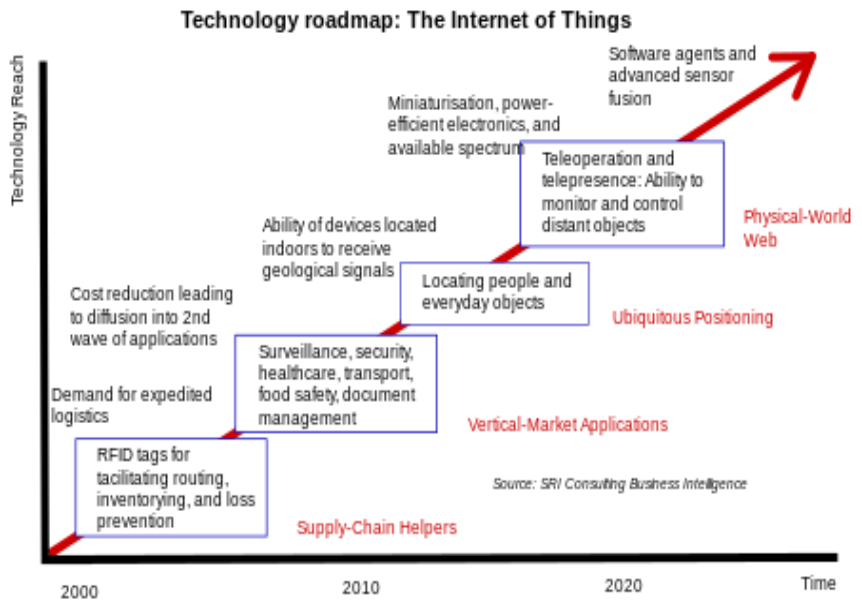

Figure 5. Technology Roadmap for The Internet of Things

Even being somewhat useful, and available now, things much more useful connected to the Internet will require the development of new products, with more sophisticated embedded sensors and computer systems designed specifically for the use of data in this way. The future with the union of two forces While the It progresses, more "smart things" will continue entering the market. Connected cars are an excellent example of the 
potential of the Internet of Things. Currently, it is not necessary for vehicles to undergo periodic maintenance checks. Instead, owners' schedule maintenance to search for possible defects or wait for a fault to occur. The flow of data from sensors on the Internet connected cars can be used to alert you when something is detected (high engine temperature, low oil, the slow response of brakes, decrease in tire pressure, etc.). The transportation industry was one of the first to invest in this technology.

Currently, operations are automatically stopped when a vehicle is faulty. In 2012, only $10 \%$ of cars in the United States were connected to the Internet, but by 2020, it is estimated that $90 \%$ of will be connected to the Internet. Other sectors are also exploring its benefits. Intel provides that local governments will spend 41 trillion dollars over the next 20 years in needed infrastructure improvements. For example, after an earthquake in 2011 that killed 185 people and severely damaged the city of Christchurch in New Zealand, disaster recovery efforts included the integration of a network of sensors connected to the city's infrastructure during its reconstruction. This is turning into a sensory Christchurch city, where the flow of Internet-connected sensor data is constantly monitored in search of air pollution, overuse of water, traffic conditions and other aspects that affect the quality of life of citizens [13]. It will take time for it to maximize its potential. However, since the network has a bigger and deeper range, the analysis of data from it may transform information into something more dynamic, giving an extra-sensory perception to detect things beyond our current capacity [14].

\section{Conclusion}

Only in more recent times, it has started to think of different solutions for access to the medium, such as, for example, the CDMA (Code Division Multiple Access). In an ad hoc it is necessary to deal with new eyes the network routing problem: it is precisely this aspect of the network that carries the major consequences of mobility and limited energy resources. Addressing these two aspects, moreover, requires opposite behavior: mobility, and therefore the dynamic topology, requires a constant exchange of information, they pursue such changes; whereas the scarcity of resources requires that this exchange is as limited as possible. The nodes, in fact, have no prior knowledge of the network topology in which there are, and they must discover it by communicating with other nodes. Generally each node announces its presence on the network and listen to the communication between other nodes, which become thus known.

As time passes each node acquires the knowledge of all network nodes and one or more ways to communicate with them. Make sure that the routing tables are reasonably small, especially in light of the limited resources of whom often have nodes in an ad hoc network. Being able to choose the best path to reach the other nodes (according to various parameters, such as speed, reliability and the absence of congestion). Keep their routing tables updated if the network topology changes, Achieve optimal operation in a short time and sending a small number of packets, possibly offer multiple paths to reach a destination, perhaps by ordering the paths in order of increasing cost. These protocols reduce the signaling overhead of purely proactive protocols, dividing the nodes into classes and maintaining an entry in the tables for each class. In this way you can reduce the signaling overhead. In detail, the network is divided into clusters on the network, where he was elected a cluster head, as is the case for the Base Station in cellular networks: one sacrifices so some of the flexibility of Ad-Hoc Network, but you get a centralized structure, which it makes it easier to manage the various aspects of communication and that also allows you to implement more scalable protocols. Within the cluster, to have always the available routes, using proactive techniques, while for inter-cluster communications use techniques reactive, in which the packets pass from a cluster end to the other until reaching the recipient cluster.

\section{References}

[1] Butt, Talal A., Provision of adaptive and context-aware service discovery for the Internet of Things, Doctoral diss., Loughborough University, Leicestershire, U.K. Ph. D., 2014

[2] Markmann, T., Securing Communications in the Internet of Things using ID-based Cryptography and Modern Elliptic Curves, Master thesis, Hamburg University of Applied Sciences, M.S., 2015

[3] Qadir, J., Ali, A., Yau, K.L.A., Sathiaseelan, A. and Crowcroft, J., Exploiting the power of multiplicity: a holistic survey of networklayer multipath, IEEE Communications Surveys \& Tutorials, 17(4), 2015, 2176-2213.

[4] Cordero, J., Yi, J., Clausen, T. and Baccelli, E., Enabling multihop communication in spontaneous wireless networks, in H. Haddadi, O. Bonaventure (Ed.), Recent Advances in Networking, (ACM SIGCOMM eBook 1 - 2013) 413-457.

[5] Detti, A., Tassetto, D., Melazzi, N.B. and Fedi, F., Exploiting content centric networking to develop topic-based, publish-subscribe MANET systems, Ad Hoc Networks, 24, 2014, 115-133.

[6] Emami, A.B., Samet, S., Azarpira, A. and Farrokhtala, A., SNACK: An efficient intrusion detection system in Mobile Ad-Hoc Network based on the Selective-Negative Acknowledgement algorithm, Proc. IEEE $28^{\text {th }}$ Canadian Conference on Electrical and Computer Engineering (CCECE), Halifax, Canada, 2015, 903-907.

[7] Khan, M.A., Khan, S., Shams, B. and Lloret, J., Distributed flood attack detection mechanism using artificial neural network in wireless mesh networks, Security and Communication Networks, 9(15), 2015, 2715-2729

[8] Oliveira, L.M., De Sousa, A.F. and Rodrigues, J.J., Routing and mobility approaches in IPv6 over LoWPAN mesh networks, International Journal of Communication Systems, 24(11), 2011, 1445-1466.

[9] Pourmohammad, S.,. Stable Queue Management in TCP/IP Networks Using Feedback Control Theory, Doctoral diss., University of Louisiana at Lafayette, USA, Ph.D, 2015 
[10] Zhao, L.,. Enhance communication security in wireless ad hoc networks through multipath routing, Doctoral diss, Washington State University, USA, Ph.D, 2007

[11] Wubshet, D., Resource Efficient Key Distribution System for MANETs, Doctoral diss, Addis Ababa University, Ethiopia, Ph.D., 2014

[12] Nilsson, A., Internet Access and Performance in Ad hoc Networks, Licenciate in Engg. diss, Lund University, Sweden, Licentiate in Engg., 2005

[13] Becker, J.S., Potter, S.H., Wein, A.M., Doyle, E.E.H. and Ratliff, J., 2015. Aftershock communication during the Canterbury earthquakes, New Zealand: Implications for response and recovery in the built environment, New Zealand Society of Earthquake Engineering Conference, Rotorua, New Zealand, 2015, 481-487

[14] Swankoski, E. and Setia, S., 2013, June. EPIC: Efficient path-independent capabilities in mobile ad hoc networks, IEEE International Workshop on Security and Privacy of Mobile, Wireless, and Sensor Networks (MWSN), 2013 